\title{
Dynamic Routing and Coordination of Cluster for Unmanned Aerial Vehicle (UAV) Swarms
}

\author{
Ali Abbas, ${ }^{1}$ Bhawani Shankar Chowdhry $\mathbb{D}^{2},{ }^{2}$ Muhammad Saqib, ${ }^{1}$ and Vishal Dattana ${ }^{1}$ \\ ${ }^{1}$ Department of Computing, Middle East College, Rusayl, Oman \\ ${ }^{2}$ NCRA-CMS Lab, Mehran University of Engineering \& Technology, Jamshoro, Pakistan
}

Correspondence should be addressed to Bhawani Shankar Chowdhry; c.bhawani@ieee.org

Received 7 July 2021; Revised 1 August 2021; Accepted 14 August 2021; Published 24 August 2021

Academic Editor: Dao B. Wang

Copyright (c) 2021 Ali Abbas et al. This is an open access article distributed under the Creative Commons Attribution License, which permits unrestricted use, distribution, and reproduction in any medium, provided the original work is properly cited.

The flying networks provide an efficient solution for a wide range of military and commercial purposes. The demand for portable and flexible communication is directed towards a quick growth in interaction among unmanned aerial vehicles (UAVs). Due to the frequent change in topology and high mobility of vehicles, routing and coordination becomes a challenging task. To maximize the throughput of the network, this study addresses the UAV swarm's problems related to the coordination and routing and defines the proposed solution to solve these issues. For this, a network is assumed which contains an equal number of dynamic vehicles. It also presents the communication graph concept of UAVs and designs a fixed-wing UAV model to improve the efficiency of the network in terms of throughput. Furthermore, the proposed algorithm based on Cauchy particle swarm optimization (CPSO) aims towards the better performance of UAV swarms and aims to solve the combinatorial problem. The simulation results show and confirm the performance of the proposed algorithm.

\section{Introduction}

In recent years, unmanned aerial vehicles (UAVs) have gained interest and have been applied in the area of search and rescue, agriculture defence, transportation, monitoring, and surveillance [1]. These vehicles can cover wider areas in the field. The coordination and monitoring system is needed for the fleet to determine their route and utilize this resource in a very efficient manner [2]. The UAV technology is also known as utilizing a network of UAVs. It attains sensing and actuating equipment to collectively perform the complicated task. Cooperation and coordination between the networks of UAV swarms will help to complete complicated missions across a large area. The decision making for the next position occurs at many stages of communication. Mostly many vehicles communicate via air to ground or air to air along with their control stations [3-5]. This technology also provides flexible configuration, improvement in the communication performance, and line-of-sight communication link on demand. Many studies show and provide a flexible solution for routing problems. A hierarchical structure is considered to have a UAV cluster. With the help of the proposed algorithm, routing takes place within the cluster and it is a basic tool for cluster communication [6]. Some other methods are used to improve the throughput of a channel by which UAV's applications expand vastly but still issues are present [7]. The UAV network information sharing is a more challenging problem limited to the throughput especially in disaster areas. It affects the overall effectiveness of the operation. For UAV swarms, different methods and techniques are designed for an optimal solution. By using these techniques, capacity of a channel is improved with an increase in the rate of the spatial multiplex.

With the development of technology, the spectrum deployment and communication ratio is highlighted. Previously, many scientists tried to solve the UAV swarm problem with different technologies, but still there are some issues to be solved. Many researchers had studied the cellular network of UAVs $[8,9]$. It is required to centralize the control due to the high intelligence level of unmanned vehicles. To address the need of UAV's network information 
distribution necessities, the challenges shall be added especially in distributed scenarios that affect the overall operation [10]. So, the effective techniques are specifically designed for UAV swarms. Cauchy particle swarm optimization (CPSO) has an effective result bearing key factors enhancing effectiveness that are topology structure, parameter selection, and swarm initialization [11, 12]. But in PSO, the disadvantage is that it easily falls into local optimum. Therefore, algorithm CPSO attains the best solution in mimimum time. The operators help in reducing the possibility of immature convergence by dealing with every ant individually.

In [13], the study provides the solution for the routing problem of the vehicle. The problem is split into a set called a cluster. This study finds the best possible distance of the route of the vehicle. The PSO technique is adopted to solve the routing issues. In [14], the improved version of PSO is called combinatorial particle swarm optimization which is used to find the number of clusters. It also increases the population variety and eliminates terminated particles. It also increases the speed of junction and solution's quality. In another study, a CPSO technique is used [15]. On the other hand, the inertia weight factor and chaotic maps are introduced to solve the clustering optimization. This study combines CPSO with weight factor and chaotic maps to attain better performance. In [16], clustering particle swarm optimization (CPSO) is proposed for the efficiency of search. This algorithm cannot guarantee convergence due to the sensitivity of the original cluster. The CPSO algorithm finds the best possible solution. Similarly, in another study [17], topology-based routing protocols improve the adeptness of the system. It also focuses on a detailed review of routing protocols that are topology-based. Furthermore, simulation evaluates the topology-based routing protocols. In [18], particle swarm optimization (PSO) with software-defined networking-based communication protocol is proposed which guarantees network management. Novel particle selection criteria are proposed for coordination purposes, which aims to guarantee network manageability of UAV formations, thus being able to guarantee service persistence in case of node failure occurrence. In [19], the article presents a review on the major research areas of unmanned vehicles, i.e., 3-degree-of-freedom (3DOF) routing algorithm and routing protocols. This article also aimed to provide a comparison between the methods and algorithms to find the best ones among these algorithms for a particular application. On the other hand, in a dynamic environment, the stability of topology is presented. The algorithm used in that article is the cluster routing algorithm used for the stability of topology and proper transmission. The proposed algorithm in [20] is paralleled with ant colony optimization (ACO) and particle swarm optimization (PSO). In [21], a new method has been proposed due to a change in the topology of a network. A dynamic routing method for a group of UAVs using SDN technology with dynamic topology was proposed. Simulation results show the comparison of the proposed method's performance with other algorithms. The simulation results show that the proposed method has better results than traditional algorithms. All these aforementioned studies use different algorithms and methods to solve the issue. But still there are issues in the topology structure, swarm initialization, and parameter selection in every algorithm. Our proposed CPSO shows effective results in solving all these issues. The only disadvantage of using PSO separately is that it falls into local optimum. So, the proposed algorithm is the best choice to solve the problem with minimum time.

Our contributions target the design of a CPSO algorithm for the better performance of UAV swarms. In this study, a swarm communication model along with a network mobility model is presented. Similarly, the fixed-wing UAV model is also presented with a communication graph concept of UAV. This article also offers the problem of UAV's swarms to maximize the throughput of the network. The network modelled is composed of multiple UAVs to form dynamic routing topologies. The advantage of the algorithm is that it improves overall fitness. Lastly, the MATLAB simulations verify the reliability, performance, and fitness of the CPSO.

This paper is organized as follows. The introduction is presented in Section 1. The state of the art is given in Section 2. The problem statement and its proposed solution are given in Section 3. Section 4 gives the preliminaries of UAV communication and its routing mobility model. In Section 5, the proposed solution is presented. The simulations are given in Section 6. Section 7 presents the conclusion of this paper.

\section{State of the Art}

A dynamic routing protocol is projected in [22] for safe and consistent transmission. The algorithm proposed in the study works on two phases that are security and routing. It finds the path which is cost-effective among the source and destination node and focuses on the security of transmitted data. In [23], a geographic position-based routing protocol for UAV networks is proposed. This is used for the dejected protocols and position-based protocols. As compared with the hop by hop routing protocols, this method fully utilizes the transmission opportunities of all the links. Similarly, in [24], UAV swarm network is proposed and the optimal number of vehicles is analysed. Moreover, a low-latency routing algorithm is designed based on the connectivity of the network and partial location information. The results verified the proposed algorithm which decreases the link average and improves the packet delivery ratio.

Likewise, in [25], a very cheap adaptive routing protocol is proposed which exploits learning algorithm and topology features for UAV swarm. This algorithm represents the most suitable parts of swarm formation which are geometric addressing module, leaf-like routing pipe, and low-complexity learning model. In [23], OLSR algorithm is proposed in terms of delay, throughput, and the load of the network. This routing protocol depends upon the link-state algorithm. Furthermore, the performance of the protocol is examined and analysed under different scenarios. The simulation results show that the link-state routing protocol has improved key indicators. Lastly, in [26], a dynamic UAV positioning method is proposed to maximize the value of sensors 
attained from multiple UAVs. To support real-time sensor data monitoring, the data should be delivered to the ground base station using an ad hoc network. In this article, particle swarm optimization is used to derive the optimum UAV location.

\section{Problem Statement and Its Solution}

3.1. Problem Definition. This section defines the problem statement and the proposed solution. Considering a given scenario having three clusters may vary according to the requirement of the task. Each of them contains an equal number of UAVs having a cluster head $(\mathrm{CH})$. Accordingly, every vehicle is required to be located at different positions or stations by communicating with each other through coordination among them. Swarms are assigned with an independent motion to form a dynamic topology. When routing and coordination of swarms takes place, the intersection points remain unchanged. The development of the flight or plan takes a little bit of time even if the inputs change concerning time. The flow comprises three steps which are as follows. (1) Start-CH, (2) $\mathrm{CH}$-Another $\mathrm{CH}$, (3) Another $\mathrm{CH}-$ End. Figure 1 shows these parts in black, red, and green colours, respectively. UAV attains flight time which is equal to the sum of the time consumed in transition between the base of fleet and interest points and elapsed time during the execution of the task at interest points. During the flight, UAV needs to share the information which is a problem to the throughput in the network.

3.2. Proposed Solution. The aforementioned problems are solved by using our proposed algorithm based on CPSO in this study. It creates an efficient strategy by fixing the parameters and different variables to reflect the current situation. It aims towards the better performance of UAV's swarms and to solve the combinatorial problem. This solution is feasible for choosing the proper cluster head and for the problem of the group of routes that may arise when the number of vehicles increases. Each route starts and ends at a station that is chosen among all available stations without overhead of the allowed time for the flight.

\section{Preliminaries of UAV Communication and Its Routing Mobility Model}

4.1. Graph Theory Concept of UAV. Assume that $G(t)=\{V(t), E(t), W(t)\}$ is a directed graph when drones are flying in a formation [27], where $V(t)=\left\{V_{1}, \ldots, V_{n}\right\}$ is called the vertex set, $E(t)$ is called the edge set, and $W(t)$ is called the weight adjacency matrix. The directed graph edge is denoted by $e_{a b}=\left(V_{a}, V_{b}\right)$, where $V_{a}$ is the tail of the edge and $V_{b}$ is the head of the edge. The weight adjacency matrix $W(t)=\left[w_{a b}\right]$, whereas the matrix elements show the adjacency weight. The communication node $a$ gets information from $b$ node when $w_{a b}>0$. The diagonal matrix is represented by $D=\operatorname{diag}\left\{d_{a} a=1, \ldots, n\right\}$ has elements of matrix $W(t)$ get added to attain $d_{a}$. Laplace matrix of graph $G(t)$ is defined as $L=D-W(t)$, where $G(t)$ is an undirected graph when $w_{a b}=w_{b a}$. The undirected graph is defined as if two nodes in the graph are connected by the edges $G(t)$. The matrix $L$ is diagonalized to its min value of matrix $\mathrm{Z}$ which satisfies

$$
0=\zeta_{1}<\zeta_{2} \leq \zeta_{\max }
$$

The eigenvalues of matrix $L$ are $\zeta_{1} \sim \zeta_{\max }$. The communication topology of UAVs consists of drones in an undirected connected graph. The second-order equation below defines the dynamic system model of UAVs.

$$
\begin{aligned}
& \left\{\begin{array}{c}
\dot{x}_{a}(t)=v_{a}(t), \\
\dot{v}_{a}(t)=c_{a}(t),
\end{array}\right. \\
& \because a=1, \ldots, n,
\end{aligned}
$$

where $x_{a}(t)$ is defined as the position status, $v_{a}(t)$ is defined as the speed status, and $c_{a}(t)$ is the control input of member $a$. The following control protocol is espoused to make sure that the formation members are consistent with the expected movement status.

$$
c_{a}(t)=\sum w_{a b}\left[n_{1}\left(x_{b}(t-T)-x_{b}(t-T)+r_{a b}\right)+n_{2}\left(v_{b}(t-T)-x_{a}(t-T)\right)\right]+\sum^{n_{3}} h_{a}\left(v_{s}-v_{a}\right)
$$

where the control gains of the system are $n_{1}, n_{2}$, and $n_{3}$. The time required for information to transfer from member $b$ to $a$ is denoted by T. $r_{a b}$ is the relative position, and $v_{s}$ is the expected speed. The ability index is denoted by $h_{a}$ which is used to attain the expected speed information of the formation. The aforementioned control protocol matrix is defined as

$$
C(t)=-n_{q}(L X(t-T)-\operatorname{diag}(W R))-n_{2} L V(t-T)-n_{3} H\left(V(t)-V_{s} \otimes 1_{k \times 1}\right),
$$

where $C(t)=\left[c_{1}(t), \ldots, c_{n}(t)\right]^{T}$ is the vector formed by the formation members control input, $x$ is comprised of position status of formation members
$X(t)=\left[x_{1}(t), \ldots ., x_{n}(t)\right]^{T}$, and the speed status vector is denoted by $V(t)=\left[v_{1}(t), \ldots, v_{n}(t)\right]^{T} . r_{p q}$ forms the matrix $R$. $\operatorname{diag}(W R)$ is a vector of $W \times R$ diagonal elements. 
Similarly, $h_{p}$ forms a diagonal matrix $H$. The systems state equation can be written as

$$
\left[\begin{array}{c}
\dot{X} \\
\dot{V}
\end{array}\right]=\left(\left[\begin{array}{ll}
0 & 1 \\
0 & 0
\end{array}\right] \otimes I_{n}-\left[\begin{array}{cc}
0 & 0 \\
0 & n_{3}
\end{array}\right] \otimes H\right)\left[\begin{array}{c}
X \\
V
\end{array}\right]-\left(\left[\begin{array}{cc}
0 & 0 \\
n_{1} & n_{2}
\end{array}\right] \otimes L\right)\left[\left[\begin{array}{c}
X(t-T) \\
V(t-T)
\end{array}\right]+\left[\begin{array}{c}
0 \\
n_{1}
\end{array}\right] \otimes \operatorname{diag}(W R)+\left[\begin{array}{c}
0 \\
n_{3} v_{s}
\end{array}\right] \otimes\left(H \times I_{k \times 1}\right)\right] .
$$

The control protocol of the above system is

$$
\left\{\begin{array}{l}
\lim _{t \rightarrow \infty}\left\|x_{a}(t)-x_{b}(t)\right\| \longrightarrow r_{a b}, \\
\lim _{t \longrightarrow \infty}\left\|v_{a}(t)-v_{b}(t)\right\| \longrightarrow 0 \\
\lim _{t \rightarrow \infty} v_{a}(t)=\lim _{t \rightarrow \infty} v_{b}(t) \longrightarrow v_{s}
\end{array}\right.
$$

The speed of both members $a$ and $b$ reached the expected speed $v_{s}$. Similarly, the relative position of both members reached the expected position $r_{a b}$.

4.2. Swarm Communication and Mobility Model. Suppose a network of UAVs forms a swarm. A swarm includes a set of aerial vehicles that can fly together and perform complicated tasks with little changes in topology. Thus, routing and coordination of swarm takes place. Similarly, swarms form a dynamic topology due to the assignment of independent motion trajectories. So, for internal swarm communication, routing is used. The network structure has a human in loop and self-organization control with some other features $[28,29]$. Furthermore, assume a control station (CS) that is answerable for providing communication to clusters. All vehicles are equipped with an antenna. A hierarchal structure network is designed. The proposed technique is implemented due to its high performance, high signal processing complexity, and so on. In the swarm communication framework, one tier is for inner and the other is for outer communication [30-32]. According to the requirement of the mission, the vehicles are divided into subgroups for swarms. The subgroup adopts aerial vehicle (AV) which is used for sharing intelligence. The structure of the cluster adopts a cluster head member (CHM). The information flow is defined in the problem statement with the help of a diagram. The communication process of swarms especially for inner and outer communication among the subgroups of swarms takes a little bit of time. Each vehicle operates in two types of mode: out of the band and in-band full-duplex. CS wirelessly communicates with $\mathrm{AV}$ and $\mathrm{CHM}$ in the same period. The swarm's communication is controlled by the clustering of CS assuming time-division mode activated by CS. Figure 2 shows the system model of swarms in which blue and green arrows show the frequencies. Similarly, orange lines show some interloping among the aerial vehicles.
4.3. Fixed-Wing UAV Model and Its Coordination. The point mass system is used to model fixed-wing UAVs [33]. Consider a swarm of UAVs given below:

$$
\begin{aligned}
& \dot{x}_{n}=V_{n} C \Gamma_{n} C \Upsilon_{n}, \\
& \dot{y}_{n}=V_{n} C \Gamma_{n} S \Upsilon_{n}, \\
& \dot{o}_{n}=V_{n} S \Gamma_{n}, \\
& V_{n}=\frac{\tau_{n}-d_{n}}{M_{n}}-g S \Gamma_{n}, \\
& \dot{\Gamma}_{n}=\frac{l_{n} S \phi_{n}-M_{n} g C \Gamma_{n}}{M_{n} V_{n} C \Gamma_{n}}, \\
& \dot{\Upsilon}_{n}=\frac{l_{n} S \phi_{n}}{M_{n} V_{n} C \Gamma_{n}},
\end{aligned}
$$

where the forward displacement and horizontal displacement are denoted by $x_{n}$ and $y_{n}$, respectively, $\dot{o}_{n}$ is the height, $V_{n}$ is the ground velocity, $\Gamma_{n}$ is the flying path angle, $\Upsilon_{n}$ is the heading angle, and $\phi_{n}$ is the banking angle. Thrust is denoted by $\tau_{n}, d_{k}$ is the drag, lift is denoted by $l_{n}$, and the gravitational acceleration and mass are denoted by $g$ and $M_{n}$, respectively. $C$ and $S$ denote sin and cos. The nonlinear model [34] by using feedback linearization can be linearized as

$$
\left\{\ddot{x}_{n}=c_{x_{n}}, \ddot{y}_{n}=c_{y_{n}}, \ddot{o}_{n}=c_{o_{n}},\right.
$$

where the simulated control inputs are $c_{x_{n}}, c_{y_{n}}$, and $c_{o_{n}}$. The actual control inputs are thrust, lift, and banking angle which can be written as

$$
\begin{gathered}
\tau_{n}=M_{n}\left[\left(c_{o_{n}}+g\right) S \Gamma_{n}+\left(c_{x_{n}} C \Upsilon_{n}+c_{y_{n}} S \Upsilon_{n}\right) C \Gamma_{n}\right]+d_{n}, \\
l_{n}=\frac{M_{n}\left(c_{o_{n}}+g\right) C \Gamma_{n}-\left(c_{x_{n}} C \Upsilon_{n}+c_{y_{n}} S \Upsilon_{n}\right) S \Upsilon_{n}}{C \phi_{n}} \\
\phi_{n}=\tan ^{-1}\left(\frac{c_{y_{n}} C \Upsilon_{n}-c_{x_{n}} S \Upsilon_{n}}{\left(c_{o_{n}}+g\right) C \Gamma_{n}-\left(c_{x_{n}} C \Upsilon_{n}+c_{y_{n}} S \Upsilon_{n}\right) S \Upsilon_{n}}\right) .
\end{gathered}
$$

Furthermore, $V_{n}, \Upsilon_{n}$, and $o_{n}$ must meet the parameters which are as follows: 


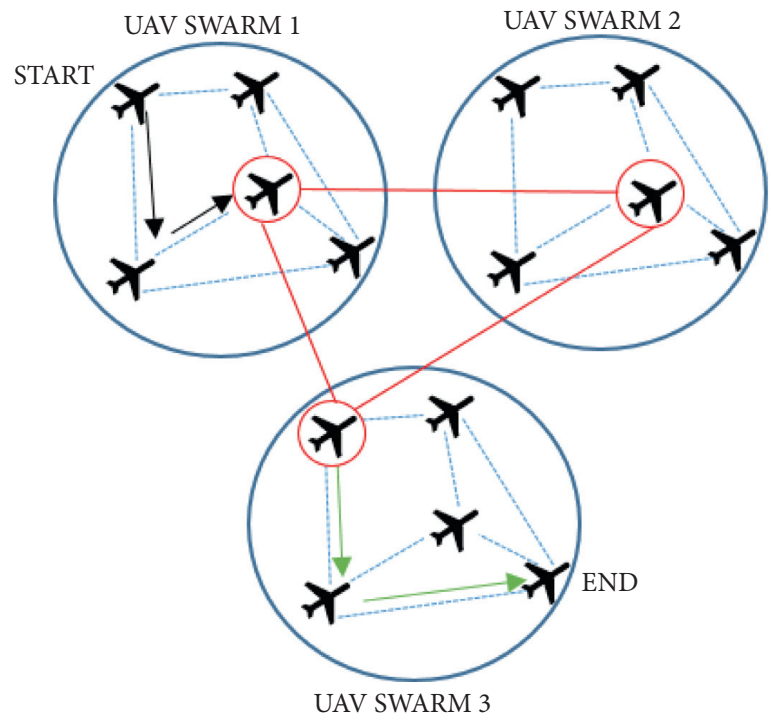

FiguRE 1: Information flow from start to endpoint.

$$
\left\{\begin{array}{c}
V_{\min } \leq V_{n} \leq V_{\max } \\
\Upsilon_{n} \leq \frac{\mu_{\max } g}{V_{n}} \\
\zeta_{\min } \leq o_{n} \leq \zeta_{\max }
\end{array}\right.
$$

where the minimum velocity and maximum velocity are denoted by $V_{\min }$ and $V_{\max }$. Maximum lateral overload is represented by $\mu_{\max }$. The minimum climbing speed and maximum climbing speed are denoted by $\zeta_{\min }$ and $\zeta_{\max }$. Two coordinate coefficients are introduced that must be added in every UAV as a function for its route to meet spatial and temporal constraints for the group. The function is expressed as

$$
\begin{aligned}
& F_{\mathbb{C}_{n}}=F_{\mathbb{T}_{n}} F_{\mathbb{S}_{n}}, \\
& F_{\mathbb{T}_{n}}=\left\{\begin{array}{l}
\frac{J}{\left(t_{1}-t_{2}\right)}, \\
0,
\end{array}\right. \\
& F_{\mathbb{S}_{n}}=\left\{\begin{array}{l}
d, \\
0,
\end{array}\right.
\end{aligned}
$$

where the coefficients of temporal and spatial coordinates are denoted by $F_{\mathbb{T}_{n}}$ and $F_{\mathbb{S}_{n}}$, respectively. $t_{1}$ and $t_{2}$ represent the expected arrival time of UAVs and $J$ is a constant. $t$ may vary depending upon the number of vehicles.

\section{Proposed Algorithm}

5.1. Cauchy Particle Swarm Optimization (CPSO). This computation-based optimization is based on the study of natural behaviours. This technique provides random solutions and updates the velocity and position to attain the universal best solution. Each unit updates the searching position with the help of its best outcome. The updated position of the unit depends upon the present velocity and past position. Now, the new position becomes the best individual position and it is superior to all other positions. The equation below becomes Cauchy distribution if $w$ meets the condition in the equation

$$
F\left(w, w_{0}, \delta\right)=\frac{1}{\pi}\left[\frac{\delta}{\left(w-w_{0}\right)^{2}+\delta^{2}}\right],
$$

whereas $w \varepsilon(-\infty,+\infty)$, the highest value of the function is denoted by $w_{0}$, the width related to half of $w_{0}$ is $\delta$. When $w_{0}$ and $\delta$ are 0 and 1, respectively, $w$ meets the probability density function (PDF) and the above equation becomes

$$
f(w, 0,1)=\frac{1}{\pi} \tan ^{-1} w+0.5 .
$$

Map operators and compass operators are used to find the global best outcome during search problems in the PSO algorithm. Therefore, a particle's position and velocity are to be determined by the outcome. Cauchy operator $K_{1}$ increases the searching area and also avoids falling into local optimum. Operator $K_{1}$ can be written as

$$
\left\{\begin{array}{l}
\frac{1}{\pi} \tan ^{-1} K_{1}+0.5 \\
K_{1}=\tan [\pi(\text { rand }-0.5)],
\end{array}\right.
$$

where rand is 0 or 1 . The rule that updates each particle $\wp_{n}$ in every repetition is given as

$$
\wp_{n}^{\prime}=\wp_{(n 0)}+K_{1}\left(\wp_{(n 0)}-\wp_{e}^{G}\right),
$$

where the calculated position of $n$ particle is represented by $\wp_{(n 0)}$. The best global position is $\wp_{e}^{G}$, and $\wp_{n}^{\prime}$ is the position of $n$ after an update. The position of $n^{\text {th }}$ particle during the next repetition is given as 


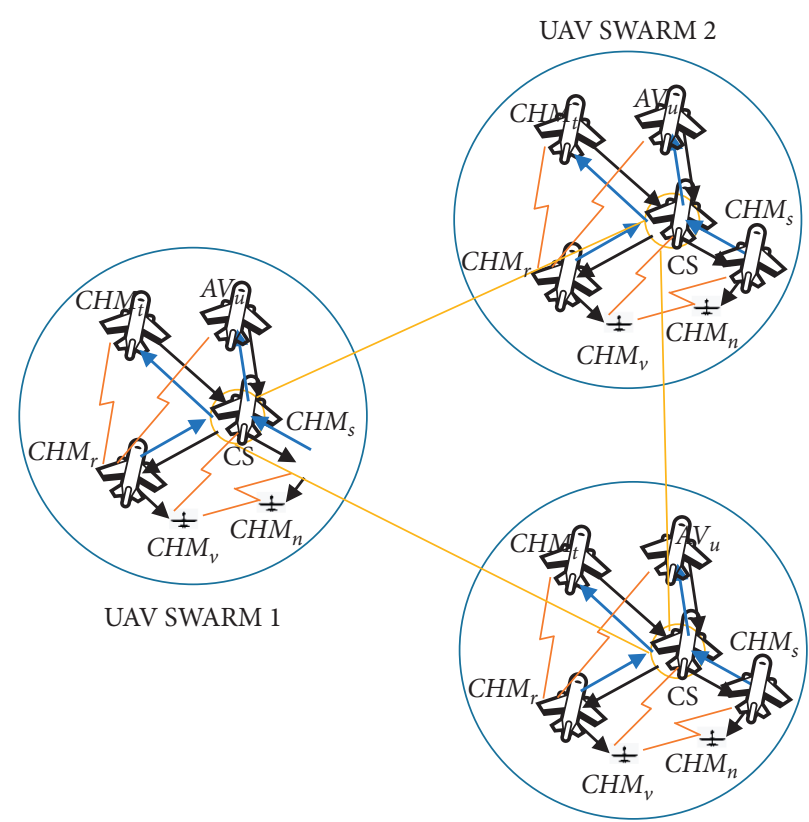

UAV SWARM 3

FIGURE 2: System model for UAV network consisting of three swarms.

$$
\wp_{n}=\left\{\begin{array}{l}
\wp_{n}^{\prime}, F\left(\wp_{(n 0)}\right)>F\left(\wp_{n}^{\prime}\right), \\
\wp_{n 0}^{\prime}, F\left(\wp_{n}^{\prime}\right)>F\left(\wp_{(n 0)}\right),
\end{array}\right.
$$

where $\wp_{n}^{\prime}$ will be far away from $\wp_{e}^{G}$ when $K_{1}$ is positive and vice versa. The most important advantage of using Cauchy operators is that particles scatter away from the center to find a better position. By equating updated position with earlier one, better fitness remains.

During the landmark operator phase, converging to the center of the swarm decreases swarm population. This problem is prevented by replacing the landmark operator with Cauchy which is used to update the particle based on its best position. The function of Cauchy is written as

$$
f(w, 0,1)=\frac{2}{\pi} \tan ^{-1} w
$$

where $w \in(0,+\infty)$. Cauchy operator $K_{2}$ is written as

$$
\left\{\begin{array}{l}
\frac{2}{\pi} \tan ^{-1} K_{2}=\text { rand } \\
K_{2}=\tan \left[\frac{2}{\pi} \text { rand }\right] .
\end{array}\right.
$$

Each particle gets updated after each repetition according to the following rule:

$$
\wp_{n 0}^{\sigma_{\min }}=\wp_{n 0}^{\sigma_{\max }}+K_{2}\left(\wp_{e}^{G}-\wp_{n 0}^{\sigma_{\max }}\right)
$$

where the position of $n^{\text {th }}$ particle is $\wp_{n 0}^{\sigma_{\max }}$. The best global position is given as $\wp_{e}^{G}$. Due to $K_{2}$, each particle moves towards the best global outcome. This operator guarantees the steady convergence of the algorithm.

Consider planning in three-dimensional space where the waypoint of each particle is $D$, position is $\wp_{n}$, and velocity vector is $V_{n}$ for $n^{\text {th }}$ particle as follows:

$$
\begin{aligned}
\wp_{n} & =\left[\wp_{(n, 1)}, \ldots, \wp_{(n, D)}\right]^{T} \\
& =\left[\left(\wp^{x}, \wp^{y}, \wp^{z}\right)_{(n, 1)}, \ldots,\left(\wp^{x}, \wp^{y}, \wp^{z}\right)_{(n, D)}\right]^{T}, \\
V_{n} & =\left[V_{(n, 1)}, \ldots, V_{(n, D)}\right]^{T} \\
& =\left[\left(V^{x}, V^{y}, V^{z}\right)_{(n, 1)}, \ldots,\left(V^{x}, V^{y}, V^{z}\right)_{(n, D)}\right]^{T},
\end{aligned}
$$

where the $q^{\text {th }}$ waypoint position and velocity of $n^{\text {th }}$ particle in space are represented as $q \in 1, \ldots, D ;\left(\wp^{x}, \wp^{y}, \wp^{z}\right)_{(n, q)}$, $\left(V^{x}, V^{y}, V^{z}\right)_{(n, q)}$. The swarm is given as if total particles in it are $P$.

$$
\left[\left(\wp_{1}, V_{1}\right), \ldots,\left(\wp_{P}, V_{P}\right)\right]
$$

In this algorithm having particles $P$, there is one global position and another individual best position $e$ which are given as

$$
\left\{\begin{aligned}
\wp_{(n, e)} & =\left[\wp_{(n, 1, e)}, \ldots, \wp_{(n, D, e)}\right]^{T}=\left[\left(\wp^{x}, \wp^{y}, \wp^{z}\right)_{(n, 1, e)}, \ldots,\left(\wp^{x}, \wp^{y}, \wp^{z}\right)_{(n, D, e)}\right]^{T} \\
\wp_{e}^{G} & =\left[\wp_{(1, e)}, \ldots, \wp_{(D, e)}\right]^{T}=\left[\left(\wp^{x}, \wp^{y}, \wp^{z}\right)_{(1, e)}, \ldots,\left(\wp^{x}, \wp^{y}, \wp^{z}\right)_{(D, e)}\right]^{T}
\end{aligned}\right.
$$


where $n \in 1, \ldots, P$ is the number of particles. Furthermore, for multi-swarm function:

$$
\begin{aligned}
& \wp_{(n, e)}(t+1)=\left\{\begin{array}{l}
\wp_{(n, e)}(t) ; \text { iff } f\left(\wp_{(n, e)}(t)\right) \leq F\left(\wp_{n}(t+1)\right), \\
\wp_{n}(t+1) ; \text { iff } f\left(\wp_{(n, e)}(t)\right)>F\left(\wp_{n}(t+1)\right),
\end{array}\right. \\
& \wp_{e}^{G} \in\left(\wp_{(n, 1, e)}, \ldots, \wp_{(n, D, e)}\right), \\
& F\left(\wp_{e}^{G}(t)\right)=\min \left[F\left(\wp_{(e, 1)}(t)\right), \ldots, F\left(\wp_{(e, D)}(t)\right)\right] .
\end{aligned}
$$

In a swarm, individual particle updates according to the following equation:

$$
\left\{\begin{array}{l}
\wp_{(n, q)}(t+1)=\wp_{(n, q)}(t)+V_{(n, q)}(t+1), \\
V_{(n, q)}(t+1)=\sigma \cdot V_{(n, q)}(t)+A_{1} B_{1} \cdot\left(\wp_{(n, q, e)}(t)-\wp_{(n, q)}(t)\right)+A_{2} B_{2} \cdot\left(\wp_{(G, q)}^{e}(t)-\wp_{(n, q)}(t)\right),
\end{array}\right.
$$

where the accelerating coefficients are $A_{1}$ and $A_{2}$ and $B_{1}$ and $B_{2}$ are two random variables which are 0 and 1 . By modifying the inertia weight $\sigma$, it can be improved, and it stabilizes the global and local outcome through search procedure. Global search can be enhanced by increasing $\sigma$. To enhance local search, decrease $\sigma$, which is given as

$$
\sigma=\frac{\left(\sigma_{\max }-\sigma_{\min }\right) t}{W},
$$

where the current repetition is $t$, maximum repetition is $W$, and $\sigma_{\min }$ and $\sigma_{\max }$ signify the minimum and maximum values of $\sigma$.

\section{Performance Evaluation}

This section of the article weighs the enactment of the proposed CPSO with the help of simulations in MATLAB. The proposed algorithm is compared with PSO and evaluated. Table 1 presents the comparison of both algorithms in terms of the mean error for localization, cost, and efficiency. There is a total of 100 nodes having max and min errors. It clearly shows that the CPSO has attained an efficient output. The conclusion is that when nodes increase, the performance of CPSO also increases. The cost value for each particle is also presented which reveals the approach which still performs the best.

6.1. Mean Error for Localization (MEFL). The simulation output figure below shows the mean error for localization with several nodes. Figure 3 clearly shows that in PSO, the mean error for localization decreases as the number of nodes increases. But in CPSO, the situation is the opposite. The proposed algorithm shows very low localization errors. The advantage of having a large number of nodes is that it localizes the node with very low error.

6.2. Accuracy ofCPSO. The accuracy in the percentage of the proposed algorithm CPSO is shown in Figure 4 along with the number of UAV nodes. It is clearly shown from the simulation output that when the number of nodes increases, the CPSO attains a higher accuracy. Both the algorithms attain a higher accuracy due to a suitable number of adjacent nodes. Our proposed algorithm has an accuracy of about 98\% when several nodes are at their maximum value.

6.3. Information Delivery Ratio (IDR). Figure 5 provides a comparison of IDR with the number of UAVs and shows the effectiveness of CPSO in delivering data of approximately more than $85 \%$. The proposed algorithm shows the best performance as compared to PSO. At some points, the ratio of both algorithms is the same, but when the number of vehicles increases, the ratio of the proposed algorithm also increases, so they have a direct relationship with each other as shown.

6.4. Average End-to-End Delay. Figure 6 shows the evaluation of average end-to-end delay with the number of UAVs of both algorithms. The simulation output clearly shows that CPSO has significantly less average end-to-end delay as compared to PSO. In high mobility networks, the delay increases because time is mandatory to find the node position. Whenever the nodes move rapidly, there is more route breakage.

6.5. Cluster Making Period (CMP). CMP is defined as the time necessary to form a cluster by the algorithm. It takes time to make a cluster generally owing to the complexity of the algorithm. Whenever a CMP is greater, the energy consumption is very high as UAVs consume high energy. As shown in Figure 7, CPSO significantly takes less time to form a cluster as compared to PSO. It is clearly shown that when no nodes increase, the proposed algorithm works proficiently and takes less time which increases its efficiency. CPSO performs clustering much better than PSO. The search space of the proposed algorithm is reduced which affects the selection of node and CMP. As compared to many algorithms, CPSO takes a short period to obtain the solution needed. In conclusion, the energy and delay decrease with the decrease in CMP. 
TABLE 1: Comparison of both algorithms in terms of localization error, cost, and efficiency.

\begin{tabular}{lcccccc}
\hline Method & $\begin{array}{c}\text { Total number of } \\
\text { nodes }\end{array}$ & $\begin{array}{c}\text { Max error for } \\
\text { localization }\end{array}$ & $\begin{array}{c}\text { Min error for } \\
\text { localization }\end{array}$ & $\begin{array}{c}\text { Mean error for } \\
\text { localization }\end{array}$ & $\begin{array}{c}\text { Mean } \\
\text { cost }\end{array}$ & $\begin{array}{c}\text { Efficiency } \\
(\%)\end{array}$ \\
\hline CPSO & 100 & 1.25 & 0.60 & 0.925 & 14.87 & 98 \\
PSO & 100 & 1.58 & 0.88 & 2.02 & 20.87 \\
\hline
\end{tabular}

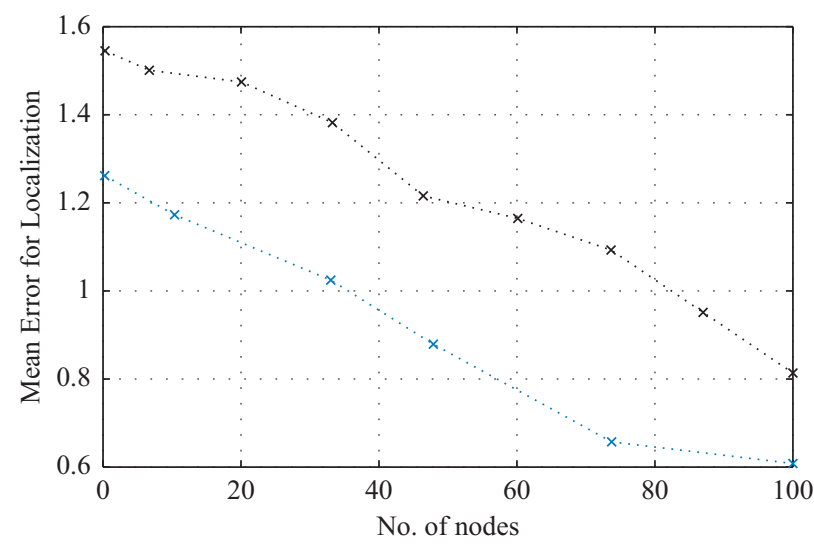

*.. PSO

*. CPSO

Figure 3: MEFL vs number of nodes.

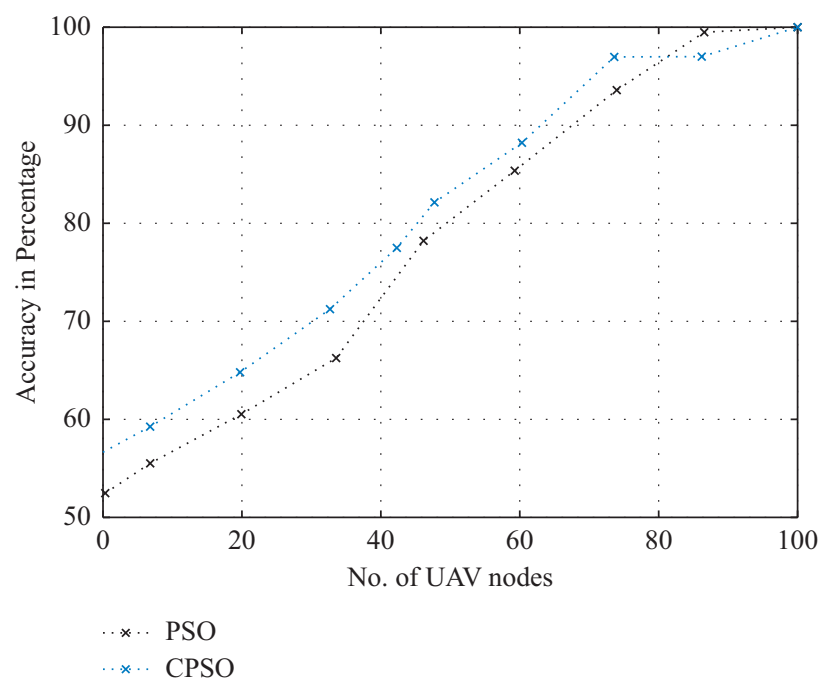

FIGURE 4: Accuracy in percentage vs number of UAV nodes. 


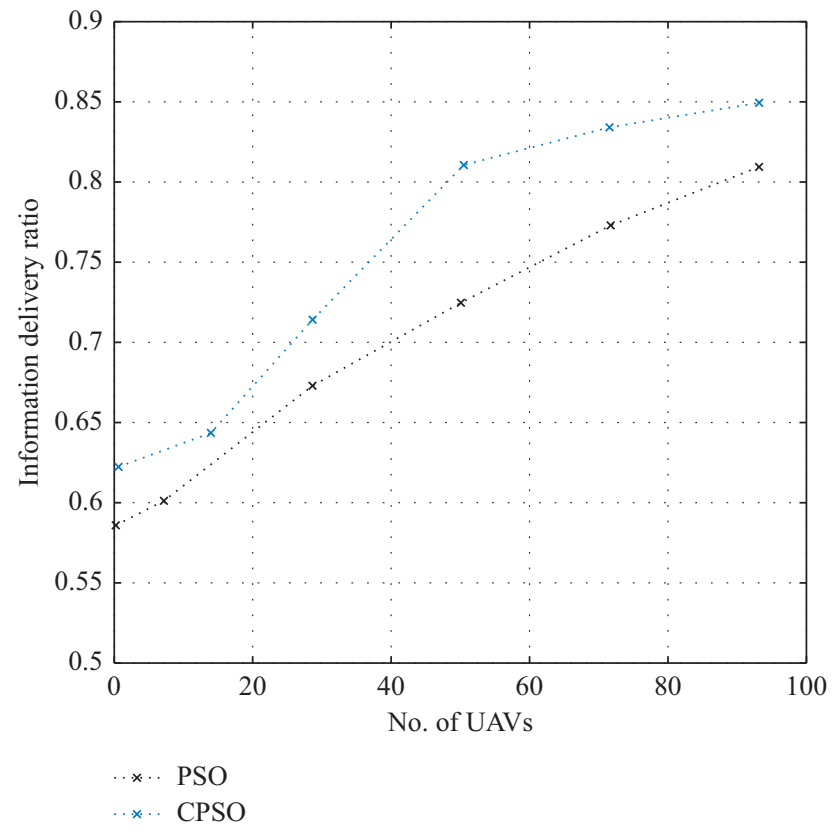

Figure 5: IDR vs number of UAVs.

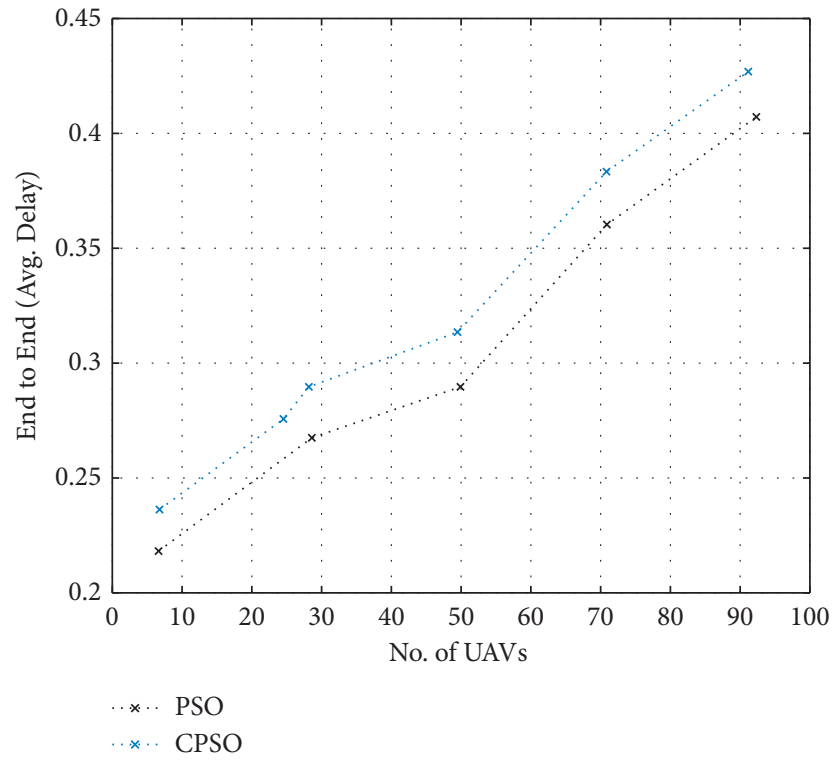

FIgURE 6: End-to-end delay vs number of UAVs. 


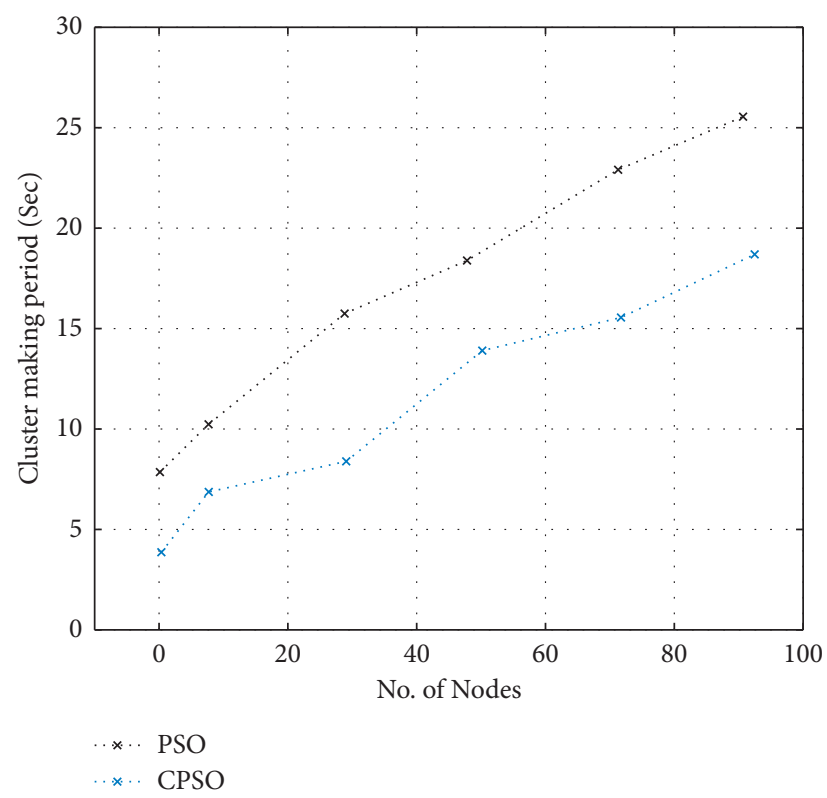

Figure 7: CMP vs number of nodes.

\section{Conclusion}

Designing an algorithm is complicated due to the dynamic topology changes and high mobility of the UAV network. This study presents the CPSO algorithm for UAV network communication which includes the coordination and routing among them. This algorithm is applied to solve node localization of UAVs in space and to solve combinational issues. It also reduces the combinational time and improves the performance of the swarms. The proposed algorithm has a convergence ability, reduces the localization error and endto-end delay, and improves the accuracy and information delivery ratio. The simulation results show the performance of the proposed algorithm along with PSO. As a result, it is seen that CPSO is suitable for better performance in communication. CPSO has a greater ability to solve all the issues. Lastly, the overall fitness of the algorithm is increased. In the future, different algorithms based on AI will be studied to achieve high-speed localization and will be applied to solve different issues.

\section{Data Availability}

The data used to support the findings of this study are included within the article.

\section{Conflicts of Interest}

The authors declare that they have no conflicts of interest.

\section{Authors' Contributions}

Ali Abbass was responsible for manuscript writing, data curation, and software. Vishal Dattana supervised the study, theorized this research, reviewed and edited the manuscript, and validated the study. Muhammad Saqib was responsible for software, data curation, and analysis. Bhawani Shankar
Chowdhry revised and edited the manuscript and examined the study.

\section{References}

[1] M. N. Boukoberine, Z. Zhou, and M. Benbouzid, "A critical review on unmanned aerial vehicles power supply and energy management: solutions, strategies, and prospects," Applied Energy, vol. 255, Article ID 113823, 2019.

[2] G. Tuna, B. Nefzi, and G. Conte, "Unmanned aerial vehicleaided communications system for disaster recovery," Journal of Network and Computer Applications, vol. 41, pp. 27-36, 2014.

[3] M. K. Oganne, Unmanned aerial vehicle swarm synchronization protocol, Ph.D. Dissertation, Botswana International University of Science \& Technology (BIUST), Palapye, Botswana, 2018.

[4] Li Wang, "Optimize 2-D path planning of mobile robot by using ACO with guidance factor," Sir Syed University Research Journal of Engineering \& Technology, vol. 9, no. 2, 2019.

[5] İ. Bekmezci, O. K. Sahingoz, and Ş. Temel, "Flying ad-hoc networks (FANETs): a survey," Ad Hoc Networks, vol. 11, no. 3, pp. 1254-1270, 2013.

[6] B. Li, Z. Fei, and Y. Zhang, "UAV communications for 5G and beyond: recent advances and future trends," IEEE Internet of Things Journal, vol. 6, no. 2, pp. 2241-2263, 2018.

[7] S. J. Nawaz, S. K. Sharma, S. Wyne, M. N. Patwary, and M. Asaduzzaman, "Quantum machine learning for $6 \mathrm{G}$ communication networks: state-of-the-art and vision for the future," IEEE Access, vol. 7, pp. 46317-46350, 2019.

[8] N. Patwari, A. O. Hero, M. Perkins, N. S. Correal, and R. J. O’Dea, "Relative location estimation in wireless sensor networks," IEEE Transactions on Signal Processing, vol. 51, no. 8, pp. 2137-2148, 2003.

[9] S. Gezici, Z. Tian, G. B. Giannakis et al., "Localization via ultra-wideband radios: a look at positioning aspects for future sensor networks," IEEE Signal Processing Magazine, vol. 22, no. 4, pp. 70-84, 2005. 
[10] R. T. Vetter, Small unmanned aircraft system integration into the Mode C Veil using an enterprise architecting framework, Ph.D. Dissertation, Massachusetts Institute of Technology, Cambridge, MA, USA, 2019.

[11] Y. Zhang, S. Wang, and G. Ji, "A comprehensive survey on particle swarm optimization algorithm and its applications," Mathematical Problems in Engineering, vol. 2015, Article ID 931256, 13 pages, 2015.

[12] Q. Guo, Y. Zhang, J. Lloret, B. Kantarci, and W. K. G. Seah, “A localization method avoiding flip ambiguities for microUAVs with bounded distance measurement errors," IEEE Transactions on Mobile Computing, vol. 18, no. 8, pp. 17181730, 2019.

[13] S. R. Venkatesan, D. Logendran, and D. Chandramohan, "Optimization of capacitated vehicle routing problem using PSO," International Journal of Engineering Science and Technology, vol. 3, no. 10, pp. 7469-7477, 2011.

[14] H. Masoud, S. Jalili, and S. M. H. Hasheminejad, "Dynamic clustering using combinatorial particle swarm optimization," Applied Intelligence, vol. 38, no. 3, pp. 289-314, 2013.

[15] C. Li, J. Zhou, P. Kou, and J. Xiao, "A novel chaotic particle swarm optimization based fuzzy clustering algorithm," Neurocomputing, vol. 83, pp. 98-109, 2012.

[16] C. Gu and T. Qian, "Clustering algorithm combining CPSO with K-Means," in Proceedings of the International Conference on Advances in Mechanical Engineering and Industrial Informatics, Zhengzhou, China, April 2015.

[17] S. Rosati, K. Krużelecki, G. Heitz, D. Floreano, and B. Rimoldi, "Dynamic routing for flying ad hoc networks," IEEE Transactions on Vehicular Technology, vol. 65, no. 3, pp. 1690-1700, 2015.

[18] T. D. Silva, "Formation coordination and network management of UAV networks using particle swarm optimization and software-defined networking, Master thesis," Universidade Federal Do Rio Grande Do Sul, Escola De Engenharia Programa De Pós-Graduação Em Engenharia Elétrica, Porto Alegre, Brazil, 2020.

[19] S. B. Amarat and Z. Peng, "3D path planning, routing algorithms and routing protocols for unmanned air vehicles: a review," Aircraft Engineering and Aerospace Technology, 2019.

[20] F. Aadil, W. Ahsan, Z. U. Rehman, P. A. Shah, S. Rho, and I. Mehmood, "Clustering algorithm for internet of vehicles (IoV) based on dragonfly optimizer (CAVDO)," The Journal of Supercomputing, vol. 74, no. 9, pp. 4542-4567, 2018.

[21] Z. A. Ali, Z. Han, and R. J. Masood, "Collective motion and self-organization of a swarm of UAVs: a cluster-based architecture," Sensors, vol. 21, no. 11, 3820 pages, 2021.

[22] V. Bhardwaj and N. Kaur, "SEEDRP: a secure energy efficient dynamic routing protocol in fanets," Wireless Personal Communications, pp. 1-27, 2021.

[23] X. Pang, M. Liu, Z. Li, B. Gao, and X. Guo, "Geographic position based hopless opportunistic routing for UAV networks," Ad Hoc Networks, vol. 120, Article ID 102560, 2021.

[24] Q. Zhang, M. Jiang, Z. Feng, W. Li, W. Zhang, and M. Pan, "IoT enabled UAV: network architecture and routing algorithm," IEEE Internet of Things Journal, vol. 6, no. 2, pp. 3727-3742, 2019.

[25] N. Toorchi, F. Hu, and S. Kumar, "Skeleton-based swarm routing (SSR): intelligent smooth routing for dynamic UAV networks," IEEE Access, vol. 9, 2020.

[26] M. Shafiq, Z. A. Ali, and E. H. Alkhammash, "A cluster-based hierarchical-approach for the path planning of swarm," Applied Sciences, vol. 11, no. 15, 6864 pages, 2021.
[27] X. Dong, Y. Li, C. Lu, G. Hu, Q. Li, and R. Zhang, "Timevarying formation tracking for UAV swarm systems with switching directed topologies," IEEE transactions on neural networks and learning systems, vol. 30, no. 12, pp. 3674-3685, 2018.

[28] V. Sharma and R. Kumar, "Cooperative frameworks and network models for flying ad hoc networks: a survey," Concurrency and Computation: Practice and Experience, vol. 29, no. 4, e3931 pages, 2017.

[29] A. Abbas, B. Shah, and K.-I. Kim, "Adaptive forwarding scheme for bounded time constraint in delay tolerant networks," Wireless Personal Communications, vol. 96, no. 2, pp. 1803-1817, 2017.

[30] I. U. Khan, "Intra-net cognitive radio intelligent utility maximization using adaptive PSO-gradient algorithm," Sir Syed University Research Journal of Engineering \& Technology, vol. 10, no. 2, 2020.

[31] M. Y. Arafat and S. Moh, "Location-aided delay tolerant routing protocol in UAV networks for post-disaster operation," IEEE Access, vol. 6, pp. 59891-59906, 2018.

[32] J. RejinaParvin and C. Vasanthanayaki, "Particle swarm optimization-based clustering by preventing residual nodes in wireless sensor networks," IEEE Sensors Journal, vol. 15, no. 8, pp. 4264-4274, 2015.

[33] C. Kang and C. A. Woolsey, "Model-based path prediction for fixed-wing unmanned aircraft using pose estimates," Aerospace Science and Technology, vol. 105, Article ID 106030, 2020.

[34] H. Zhao, J. Liu, and D. Yu, “Approximate nonlinear modeling and feedback linearization control for aeroengines," Journal of Engineering for Gas Turbines and Power, vol. 133, no. 11, 2011. 